\title{
Analysis of Combining Ability and Gene Action in Indian bean [Lablab purpureus (L.) Sweet]
}

\author{
Shreya Sen*, R.L. Patel, R.A. Patel, K.G. Modha and R.K. Patel \\ Department of Genetics and Plant Breeding, N. M. College of Agriculture, Navsari \\ Agricultural University, Navsari, Gujarat, India \\ *Corresponding author
}

\section{Key words \\ Heterosis, Combining ability, Transgressive segregants}

\section{Article Info}

Accepted:

16 March 2018

Available Online:

10 April 2018

\section{A B S T R A C T}

An experiment was conducted involving 10 diverse parents and their 24 hybrids obtained by line $\mathrm{x}$ tester mating design laid out in randomized block design with three replications. The analysis of variance revealed presence of ample variability for fifteen quantitative traits studied. The genotypes Katargam, GP-167, GP-158 and GP-kh-64 were found to be good general combiners for one or more yield attributes. The cross combination GP-167 x Katargam exhibited highest mean values and standard heterosis over check variety Gujarat Papadi-1 for green pod yield per plant followed by GP-167 x GNIB-21 and GP-189 x GNIB-21. These hybrids had good general combining parents, high sca effects and heterotic effects over better parent as well as standard check. Both additive and nonadditive gene actions played important role for the inheritance of most of the traits. While, only non-additive gene action was responsible for days to flowering, plant height, primary branches per plant, seeds per pod and straw yield per plant. The ratio of $\sigma^{2}$ gca $/ \sigma^{2}$ sca indicated the preponderance of non-additive gene action for all the traits except green pod yield per plant. The superior hybrids may be advanced to obtain transgressive segregants and purelines for higher green pod yield per plant. As the development of hybrid variety is not possible in Indian bean, diallel selective mating system may be adopted for improvement of remaining traits followed by some sort of biparental mating and recurrent selection.

\section{Introduction}

Indian bean [Dolichos lablab (L.), syn. Lablab purpureus (L.) Sweet], belongs to Fabaceae family $(2 \mathrm{n}=22)$, is an important pulse crop grown for its nutritious tender pods, while dry seeds are used as a split pulse. Green pod has a high nutritive value as it contains 25 per cent proteins, 2.7 per cent fats, 62.2 per cent carbohydrates, 16.1 per cent fiber and 7.1 per cent ash (Kay, 1973). It is grown as grain and green vegetables as well as fodder throughout the tropics and sub-tropics. It is considered as a minor pulse crop in India. Indian bean fits well in a variety of cropping systems such as cover crop, mix crop and catch crop as pulses have unique property of maintaining and restoring soil fertility through biological nitrogen fixation as well as conserving and improving physical properties of soil by addition of organic matter through leaf drop towards maturity and deep root system. 
Average yield of Indian bean is very low and year to year yield variation is remarkably high. Poor yield potentiality of Indian bean may be due to the genotypes adapted to poor management practices as it is constantly grown under marginal lands, residual moisture or stress conditions. It is believed that pulses may have lower genetic potential than cereals, however, the available evidences indicates that the pulse crops have higher genetic potential for yield than cereals (Jain, 1975).

Development of high yielding hybrid or improved variety is the main objective of almost all crop improvement programmes. As the research work done for genetic evaluation and improvement of Indian bean is very scanty, attempts in Indian bean varietal improvement would, therefore, need development and execution of very soundbreeding programmes. Hybridization is frequently used in any breeding programme to improve the yield. Yield is a complex genetic phenomenon which depends upon the balance of additive, dominance and epistatic components of the genetic variance. Due to this, the presence or absence of heterosis is not itself indicative of the presence or absence of any particular type of gene effects. The nature of gene effects varies with the genetic structure and the divergence between populations. Consequently, a high yielding line may not be able to transmit its superiority to its progeny. A distinct knowledge about the type of gene effect, its magnitude and probable future breeding consequences is of fundamental importance to a plant breeder.

The line $\mathrm{x}$ tester analysis technique has been extensively used as compared to other methods because it provides a more systematic approach to assess the combining ability of parents and crosses for different quantitative characters as well as to study the extent of heterosis for yield and yield contributing characters. Besides, it gives over all genetic picture of the materials under investigation in a single generation. The present investigation is, therefore, proposed to estimate combining ability of parents and nature of gene actions involved in the inheritance of yield and its components through Line $\mathrm{x}$ Tester design in Indian bean.

\section{Materials and Methods}

The experimental material for the present investigation comprised of 10 parents and their 24 hybrids developed through Line X Tester mating design. Genotypes used as lines were GP-189, GP-158, GP-KH-120, GP-16, GP-KH-64 and GP-1, and the testers were GNIB-21, Katargam, Kapasi and Manchhi. GNIB-21 is recently released variety for vegetable purpose which is determinate, pole type and photo-insensitive. While, Katargam, Kapasi and Manchhi are landraces with marketable pod qualities. Crossing programme for present investigation was carried out in Late-Kharif, 2014-15 at College Farm, N. M. College of Agriculture, Navsari Agricultural University, Navsari. $F_{1}$ seeds were produced by hand emasculation and pollen transfer. The mature dry pods from female parents were harvested cross-wise, separately. For parental lines, flower buds of different parents were selfed and seeds were collected accordingly. Evaluation of parents along with $F_{1} s$ was carried out during Late-Kharif, 2015-16 at same location for genetic parameters like combining ability and gene actions. Twenty four $\mathrm{F}_{1}$ hybrids along with 10 parents including check variety Gujarat Papadi-1 were evaluated in a randomized block design with three replications. Each plot consisted of a single row of 10 plants. Inter- and intra-row spacing was kept $90 \mathrm{~cm}$ and $45 \mathrm{~cm}$, respectively. All the recommended package of practices was adopted to raise a healthy crop.

Five random and competitive plants were selected from each row in each replication to 
record the observations. Remaining five plants were utilized to observe green pod yield per plant and related traits. The data was recorded for the characters like days to $50 \%$ flowering, days to maturity, plant height, primary branches per plant, pods per plant, pod length, pod width, pod weight, seeds per pod, green pod yield per plant, seed yield per plant, harvest index, straw yield, 100 dry seed weight and protein content. The observations on days to $50 \%$ flowering and days to maturity were recorded on population basis. The data obtained for each character was analyzed by the usual standard statistical procedure suggested by Panse and Sukhatme (1978). The variation among the hybrids was partitioned further into sources attributable to general and specific combining ability components in accordance with the procedure suggested by Kempthorne (1957) and modified by Arunachalam (1974).

\section{Results and Discussion}

The character-wise mean data of parents and $\mathrm{F}_{1} \mathrm{~S}$ were subjected to analysis of variance for green pod yield per plant and its attributes. The results of analysis of variance of parents and their hybrids for various traits are given in Table 1. Mean squares due to genotypic differences were found significant for all the traits studied which indicated that experimental material under study had sufficient genetic diversity.

Further, partitioning of sum of squares due to genotypes indicated that the differences among parents were significant for all the traits. In case of hybrids, significant differences were obtained for all the traits studied. While, mean squares due to parents vs. hybrids were significant for all the traits studied except pod length, pod weight and seeds per pods. This indicated that the hybrids were significantly different among themselves and from the parents for mentioned traits.
The genetic variances were estimated from the analysis of variance for combining ability for fifteen characters as suggested by Kempthorne (1957). The results are presented in Table 2, the estimates of combining ability of hybrids were highly significant for all the traits. Mean squares due to lines' effects were nonsignificant for pods per plant, pod length and harvest index, whereas it was highly significant for pod width, pod weight, green pod yield per plant and 100 dry seed weight. While, significant estimates of testers' mean squares were observed for days to maturity, pods per plant, pod length, pod width, green pod yield per plant and seed yield per plant.

Line $\mathrm{x}$ tester effects were also highly significant for all the characters except days to flowering, primary branches per plant and green pod yield per plant. The ratio of $\sigma^{2}$ gca / $\sigma^{2}$ sca indicated the preponderance of additive gene effects for the inheritance of green pod yield per plant. Whereas, the ratio suggested preponderance of non-additive gene actions for remaining traits. The results are in accordance with Srinivasan and Das (1996), Valu et al., (1999), Bagade (2001), Bagade et al., (2003), Vashi et al., (2001), Tukadiya et al., (2006), Modha et al., (2007), Rahman et al., (2009), Patil et al., (2013), Das et al., (2014) and Kumar et al.,(2015).

The parents were classified as good, average and poor general combiners based on estimates of general combining ability effects (Table 3). The analysis revealed that the genotype Katargam was found to be good general combiner for pods per plant, pod length, pod width, pod weight, seed yield per plant, straw yield and 100- dry seed weight; GP-167 for days to $50 \%$ flowering, plant height, pod width, pod weight, green pod yield per plant, straw yield and 100 dry seed weight; GP-158 for pod length, pod width, pod weight, seeds per pod, seed yield per plant, straw yield and 100 dry seed weight; GP-kh-64 for pods 
per plant, green pod yield per plant, seed yield per plant and harvest index; Manchhi for plant height, pod length, pod width, straw yield and 100-dry seed weight; Kapasi for pods per plant, pod weight, seed yield per plant and harvest index; GP-189 for plant height, pod length, pod weight, green pod yield per plant and straw yield; GP-1 for pods per plant, seed yield per plant and harvest index; GP-kh-120 for pods per plant, green pod yield per plant and harvest index; GNIB-21 for days to maturity, seeds per pod and green pod yield per plant Therefore, parents Katargam, GP167 and GP-158 may be utilized for future breeding programme in Indian bean. While, recently released variety GNIB-21 can be utilized for genetic improvement of earliness and green pod yield per plant (Table 4).

Table.1 Analysis of variance for various characters in Indian bean

\begin{tabular}{|c|c|c|c|c|c|c|c|c|c|c|}
\hline $\begin{array}{l}\mathbf{M} \\
\mathbf{E}\end{array}$ & $\begin{array}{l}\text { Sources of } \\
\text { variation }\end{array}$ & replication & genotypes & parents & Females & males & $\begin{array}{l}\text { Female vs } \\
\text { males }\end{array}$ & $\begin{array}{l}\text { Parents vs } \\
\text { hybrids }\end{array}$ & hybrids & error \\
\hline $\mathbf{A}$ & d.f & 2 & 33 & 9 & 5 & 3 & 1 & 1 & 23 & 66 \\
\hline $\mathbf{I V}$ & $\begin{array}{l}\text { Days to } 50 \% \\
\text { flowering }\end{array}$ & 2.14 & $32.85 * * *$ & $69.07 * * *$ & $20.32 *$ & $136.0 * * *$ & $112.0 * * *$ & $34.12 *$ & $18.62 *$ & 7.28 \\
\hline $\begin{array}{l}\mathrm{U} \\
\mathrm{M}\end{array}$ & $\begin{array}{l}\text { Days to } \\
\text { maturity }\end{array}$ & 4.32 & $222.8 * * *$ & $355.1 * * *$ & $146.3^{* * *}$ & $808.5 * * *$ & $39.20 * *$ & $23.71 *$ & $179.7 * * *$ & 4.70 \\
\hline $\mathbf{O}$ & $\begin{array}{l}\text { Plant height } \\
(\mathrm{cm})\end{array}$ & 28.73 & $284.7 * * *$ & $165.5^{* * *}$ & $134.1 * * *$ & $252.4 * * *$ & 62.18 & $952.7 * * *$ & $302.3 * * *$ & 22.43 \\
\hline $\mathbf{F}$ & $\begin{array}{l}\text { Primary } \\
\text { branches per } \\
\text { plant }\end{array}$ & 0.34 & $0.71^{* * *}$ & $1.23 * * *$ & 0.30 & $2.32 * * *$ & $2.59 * * *$ & $4.11^{* * *}$ & $0.36^{*}$ & 0.19 \\
\hline $\begin{array}{l}\mathbf{S} \\
\mathbf{Q}\end{array}$ & $\begin{array}{l}\text { Pods per } \\
\text { plant }\end{array}$ & $4090.6^{* * * *}$ & $7758.1 * * *$ & $5258.8 * * *$ & 94.21 & $11583.0 * * *$ & $12109.7^{* * *}$ & $36658.9 * * *$ & $7479.5 * * *$ & 73.53 \\
\hline $\begin{array}{l}\mathbf{U} \\
\mathbf{A}\end{array}$ & $\begin{array}{l}\text { Pod length } \\
(\mathrm{mm})\end{array}$ & $63.32 * * *$ & $427.23 * * *$ & $794.7 * * *$ & $575.9 * * *$ & $112.0 * * *$ & $3936.8 * * *$ & 0.19 & $302.2 * * *$ & 2.90 \\
\hline $\begin{array}{l}\mathbf{R} \\
\mathbf{E}\end{array}$ & $\begin{array}{l}\text { Pod width } \\
(\mathrm{mm})\end{array}$ & $5.93 * * *$ & $32.16^{* * *}$ & $48.93 * * *$ & $46.92 * * *$ & $6.99 * * *$ & $184.83 * * *$ & $66.63 * * *$ & $24.09 * * *$ & 0.67 \\
\hline$S$ & $\begin{array}{l}\text { Pod weight } \\
\text { (g) }\end{array}$ & $0.61 * * *$ & $0.95 * * *$ & $1.32 * * *$ & 1.15 & $1.41 * * *$ & $1.88 * * *$ & 0.029 & $0.85 * * *$ & 0.031 \\
\hline & $\begin{array}{l}\text { Seeds per } \\
\text { pods }\end{array}$ & 0.085 & $0.24 * * *$ & $0.20 * * *$ & $0.27 * * *$ & 0.065 & $0.33 *$ & 0.19 & $0.26^{* * *}$ & 0.057 \\
\hline & $\begin{array}{l}\text { Green pod } \\
\text { yield per } \\
\text { plant }\end{array}$ & 4.09 & $2899.5 * * *$ & $4892.0 * * *$ & $2073.3 * * *$ & $1699.8 * * *$ & $28561.6^{* * * *}$ & $12173.9 * * *$ & $1716.6^{* * *}$ & 39.6 \\
\hline & $\begin{array}{l}\text { Seed yield } \\
\text { per plant }(\mathrm{g})\end{array}$ & $4496.4^{* * *}$ & $3868.2 * * *$ & $2085.9 * * *$ & $500.6 * * *$ & $5315.9 *$ & 322.1 & $31890.8 * * *$ & $3347.3 * * *$ & 101.9 \\
\hline & $\begin{array}{l}\text { Harvest } \\
\text { index }(\%)\end{array}$ & $241.2 * * *$ & $265.3^{* * *}$ & $380.9 * * *$ & $61.8 * * *$ & $733.3 * * *$ & $919.0 * * *$ & $183.7^{* * *}$ & $223.7 * * *$ & 5.40 \\
\hline & $\begin{array}{l}\text { Straw yield } \\
\text { (g) }\end{array}$ & 83.37 & $13919.7 * * *$ & $19620.6^{* * *}$ & $15474.0 * * *$ & $26468.9 * * *$ & $19808.4 * * *$ & $40819.4 * * *$ & $10519.4 * * *$ & 75.85 \\
\hline & $\begin{array}{l}100 \text { seed } \\
\text { weight }(\mathrm{g})\end{array}$ & 0.59 & $71.55^{* * *}$ & $87.61 * * *$ & $78.13 * * *$ & $5.31 * * *$ & $381.9 * * *$ & $6.92 * * *$ & $68.08 * * *$ & 0.20 \\
\hline & $\begin{array}{l}\text { Protein } \\
\text { content }(\%)\end{array}$ & 0.62 & $36.96^{* * *}$ & $90.57 * * *$ & $108.2 * * *$ & $12.82 * * *$ & $235.5 * * *$ & $42.18 * * *$ & $15.76 * * *$ & 0.64 \\
\hline
\end{tabular}

$*$, **, *** significance at 5 per cent, 1 per cent and 0.1 percent levels of probability, respectively 
Table.2 Mean squares due to general and specific combining ability for different characters in Indian bean

\begin{tabular}{|c|c|c|c|c|c|c|c|c|c|c|c|c|}
\hline & LINES & & & & & & & TESTERS & & & & \\
\hline parents & GP -189 & GP -158 & $\begin{array}{l}\text { GP-Kh- } \\
120\end{array}$ & GP-167 & $\begin{array}{l}\text { GP-kh- } \\
64\end{array}$ & GP-1 & S.E.(gi) & $\begin{array}{l}\text { GNIB- } \\
21\end{array}$ & Katargam & kapasi & Manchhi & $\begin{array}{l}\text { S.E. } \\
\text { (gj) }\end{array}$ \\
\hline $\begin{array}{l}\text { Days to } \\
50 \% \\
\text { flowering }\end{array}$ & 0.73 & 1.23 & -0.76 & $-2.01^{*}$ & 0.81 & 0.014 & 0.77 & -0.98 & $1.79 * *$ & 0.45 & -1.26 & 0.63 \\
\hline $\begin{array}{l}\text { Days to } \\
\text { maturity }\end{array}$ & -0.87 & 0.042 & 1.12 & 0.70 & 0.12 & -1.12 & 0.62 & $-9.54 * * *$ & $2.95 * * *$ & -0.81 & $7.40 * * *$ & 0.51 \\
\hline $\begin{array}{l}\text { Plant } \\
\text { height } \\
\text { (cm) }\end{array}$ & $7.67 * * *$ & $-9.75 * * *$ & 0.95 & $5.11 * * *$ & 2.42 & $-6.42 * * *$ & 1.36 & $-2.93^{*}$ & -1.04 & 1.04 & $2.92 *$ & 1.11 \\
\hline $\begin{array}{l}\text { Primary } \\
\text { branches } \\
\text { per plant }\end{array}$ & -0.097 & -0.18 & 0.23 & -0.11 & 0.13 & 0.019 & 0.12 & -0.13 & -0.014 & 0.042 & 0.10 & 0.10 \\
\hline $\begin{array}{l}\text { Pods per } \\
\text { plant }\end{array}$ & $-47.10^{* * * *}$ & $-20.36^{* * *}$ & $32.43 * * *$ & $-15.25 * * *$ & $41.76^{* * *}$ & $8.51 * *$ & 2.47 & $-30.20 * * *$ & $13.68 * * *$ & $37.42 * * *$ & $-20.91 * * *$ & 2.02 \\
\hline $\begin{array}{l}\text { Pod } \\
\text { length } \\
(\mathrm{mm})\end{array}$ & $11.60 * * *$ & $4.76^{* * * *}$ & $-7.10 * * *$ & 0.00 & $-4.10 * * *$ & $-5.16 * * *$ & 0.49 & -0.52 & $5.11 * * *$ & $-8.22 * * *$ & $3.63 * * *$ & 0.40 \\
\hline $\begin{array}{l}\text { Pod width } \\
\text { (mm) }\end{array}$ & 0.06 & $2.66 * * *$ & $-2.16 * * *$ & $1.58 * * *$ & $-2.54 * * *$ & 0.38 & 0.23 & $-1.42 * * *$ & $1.18 * * *$ & $-1.44 * * *$ & $1.68 * * *$ & 0.19 \\
\hline $\begin{array}{l}\text { Pod } \\
\text { weight (g) }\end{array}$ & $0.36 * * *$ & $0.49 * * *$ & $-0.43 * * *$ & $0.29 * * *$ & $-0.36 * * *$ & $-0.35 * * *$ & 0.05 & $-0.24 * * *$ & 0.20 **** & $0.17 * * *$ & $-0.13 * *$ & 0.04 \\
\hline $\begin{array}{l}\text { Seeds per } \\
\text { pods }\end{array}$ & -0.042 & $0.25 * * *$ & $-0.17 * * *$ & 0.12 & $-0.19 * *$ & 0.025 & 0.06 & $0.24 * *$ & $-0.14 *$ & -0.064 & -0.042 & 0.05 \\
\hline $\begin{array}{l}\text { Green } \\
\text { pod yield } \\
\text { per plant } \\
\text { (g) }\end{array}$ & $15.38 * * *$ & $-29.43 * * *$ & $10.71 * * *$ & $27.11 * * *$ & $8.48^{* * * *}$ & $-32.26 * * *$ & 1.81 & $6.92 * * *$ & -0.45 & $-3.07^{*}$ & $-3.40^{*}$ & 1.48 \\
\hline $\begin{array}{l}\text { Seed yield } \\
\text { per plant } \\
\text { (g) }\end{array}$ & $-39.45^{* * *}$ & $10.54 * * *$ & -2.14 & 5.70 & $14.14 * * *$ & $11.20^{* * * *}$ & 2.91 & $-24.51 * * *$ & $15.63 * * *$ & $18.36^{* * *}$ & $-9.48 * * *$ & 2.38 \\
\hline $\begin{array}{l}\text { Harvest } \\
\text { index }(\%)\end{array}$ & $-12.09 * * *$ & -0.027 & $4.59 * * *$ & -1.26 & $3.68 * * * *$ & $5.11 * * *$ & 0.67 & $-1.58 * *$ & 0.75 & $4.19 * * *$ & $-3.37 * * *$ & 0.54 \\
\hline $\begin{array}{l}\text { Straw } \\
\text { yield (g) }\end{array}$ & $40.14 * * *$ & $24.87 * * *$ & $-48.11 * * *$ & $27.99 * * *$ & $-17.44 * * *$ & $-27.44 * * *$ & 2.51 & $-32.15 * * *$ & $26.79 * * *$ & $-6.78 * *$ & $12.14 * * *$ & 2.05 \\
\hline $\begin{array}{l}100 \text { seed } \\
\text { weight }\end{array}$ & -0.22 & $4.90 * * *$ & $-4.87 * * *$ & $3.55 * * *$ & $-3.32 * * *$ & -0.042 & 0.13 & $-1.39 * * *$ & $1.58 * * *$ & $-1.91 * * *$ & $1.71 * * *$ & 0.10 \\
\hline $\begin{array}{l}\text { Protein } \\
\text { content } \\
(\%)\end{array}$ & $0.85^{* * * *}$ & $0.84 * * *$ & $-3.62 * * *$ & -0.44 & $1.82 * * *$ & $0.54 * * *$ & 0.23 & $0.88 * * *$ & 0.36 & $-0.50 *$ & $-0.73 * * *$ & 0.18 \\
\hline
\end{tabular}

$*, * *, * * *$ Significant at $5 \%, 1 \%$ and $0.1 \%$ levels of probability, respectively. 
Table.3 Estimation of gca effects of parents for various characters in Indian bean

\begin{tabular}{|c|c|c|c|c|c|c|c|c|c|c|c|c|c|c|c|}
\hline & $\begin{array}{c}\text { Days to } \\
50 \% \\
\text { flowering }\end{array}$ & $\begin{array}{l}\text { Days to } \\
\text { maturity }\end{array}$ & $\begin{array}{c}\text { Plant } \\
\text { height } \\
(\text { cm) }\end{array}$ & $\begin{array}{c}\text { Primary } \\
\text { branches } \\
\text { per } \\
\text { plant }\end{array}$ & $\begin{array}{c}\text { Pods } \\
\text { per } \\
\text { plant }\end{array}$ & $\begin{array}{c}\text { Pod } \\
\text { length } \\
(\mathrm{mm})\end{array}$ & $\begin{array}{c}\text { Pod } \\
\text { width } \\
(\mathrm{mm})\end{array}$ & $\begin{array}{c}\text { Pod } \\
\text { weight } \\
(\mathrm{g})\end{array}$ & $\begin{array}{c}\text { Seeds } \\
\text { per } \\
\text { pods }\end{array}$ & $\begin{array}{c}\text { Green } \\
\text { Pod } \\
\text { yield per } \\
\text { plant (g) }\end{array}$ & $\begin{array}{c}\text { Seed } \\
\text { yield per } \\
\text { plant (g) }\end{array}$ & $\begin{array}{c}\text { Harvest } \\
\text { index } \\
(\%)\end{array}$ & $\begin{array}{c}\text { Straw } \\
\text { yield }(\mathrm{g})\end{array}$ & $\begin{array}{c}100- \\
\text { seed } \\
\text { weight } \\
(\mathrm{g})\end{array}$ & $\begin{array}{c}\text { Protein } \\
\text { content } \\
(\%)\end{array}$ \\
\hline \multicolumn{16}{|c|}{ Lines } \\
\hline GP-189 & 0.73 & -0.87 & $7.67 * * *$ & -0.097 & $-47.10 * * *$ & $11.60 * * *$ & 0.06 & $0.36 * * *$ & -0.042 & $15.38 * * *$ & $-39.45 * * *$ & $-12.09 * * *$ & $40.14 * * *$ & -0.22 & $0.85 * * *$ \\
\hline GP-158 & 1.23 & 0.042 & $-9.75 * * *$ & -0.18 & $-20.36 * * *$ & $4.76 * * *$ & $2.66 * * *$ & $0.49 * * *$ & $0.25 * * *$ & $-29.43 * * *$ & $10.54 * * *$ & -0.027 & $24.87 * * *$ & $4.90 * * *$ & $0.84 * * *$ \\
\hline $\begin{array}{l}\text { GP-kh - } \\
120\end{array}$ & -0.76 & 1.12 & 0.95 & 0.23 & $32.43 * * *$ & $-7.10 * * *$ & $-2.16 * * *$ & $-0.43 * * *$ & $-0.17 * * *$ & $10.71 * * *$ & -2.14 & $4.59 * * *$ & $-48.11 * * *$ & $-4.87 * * *$ & $-3.62 * * *$ \\
\hline GP-167 & $-2.01 *$ & 0.70 & $5.11 * * *$ & -0.11 & $-15.25 * * *$ & 0.00 & $1.58 * * *$ & $0.29 * * *$ & 0.12 & $27.11 * * *$ & 5.70 & -1.26 & $27.99 * * *$ & $3.55 * * *$ & -0.44 \\
\hline GP-kh-64 & 0.81 & 0.12 & 2.42 & 0.13 & $41.76 * * *$ & $-4.10 * * *$ & $-2.54 * * *$ & $-0.36 * * *$ & $-0.19 * *$ & $8.48 * * *$ & $14.14 * * *$ & $3.68 * * *$ & $-17.44 * * *$ & $-3.32 * * *$ & $1.82 * * *$ \\
\hline GP-1 & 0.014 & -1.12 & $-6.42 * * *$ & 0.019 & $8.51 * *$ & $-5.16 * * *$ & 0.38 & $-0.35 * * *$ & 0.025 & $-32.26 * * *$ & $11.20 * * *$ & $5.11 * * *$ & $-27.44 * * *$ & -0.042 & $0.54 * * *$ \\
\hline S.E. (gi) & 0.77 & 0.62 & 1.36 & 0.12 & 2.47 & 0.49 & 0.23 & 0.05 & 0.06 & 1.81 & 2.91 & 0.67 & 2.51 & 0.13 & 0.23 \\
\hline \multicolumn{16}{|c|}{ Testers } \\
\hline GNIB - 21 & -0.98 & $-9.54 * * *$ & $-2.93 *$ & -0.13 & $-30.20 * * *$ & -0.52 & $-1.42 * * *$ & $-0.24 * * *$ & $0.24 * * *$ & $6.92 * * *$ & $-24.51 * * *$ & $-1.58 * *$ & $-32.15 * * *$ & $-1.39 * * *$ & $0.88 * * *$ \\
\hline Katargam & $1.79 * *$ & $2.95 * * *$ & -1.04 & -0.014 & $13.68 * * *$ & $5.11 * * *$ & $1.18 * * *$ & $0.20 * * *$ & $-0.14 *$ & -0.45 & $15.63 * * *$ & 0.75 & $26.79 * * *$ & $1.58 * * *$ & 0.36 \\
\hline Kapasi & 0.45 & -0.81 & 1.04 & 0.042 & $37.42 * * *$ & $-8.22 * * *$ & $-1.44 * * *$ & $0.17 * * *$ & -0.064 & $-3.07 *$ & $18.36 * * *$ & $4.19 * * *$ & $-6.78 * *$ & $-1.91 * * *$ & $-0.50 *$ \\
\hline Manchhi & -1.26 & $7.40 * * *$ & $2.92 *$ & 0.10 & $-20.91 * * *$ & $3.63 * * *$ & $1.68 * * *$ & $-0.13 * *$ & -0.042 & $-3.40 *$ & $-9.48 * * *$ & $-3.37 * * *$ & $12.14 * * *$ & $1.71 * * *$ & $-0.73 * * *$ \\
\hline S.E. (gj) & 0.63 & 0.51 & 1.11 & 0.10 & 2.02 & 0.40 & 0.19 & 0.04 & 0.05 & 1.48 & 2.38 & 0.54 & 2.05 & 0.10 & 0.18 \\
\hline
\end{tabular}

$*$, **, *** Significant at $5 \%, 1 \%$ and $0.1 \%$ levels of probability, respectively. 
Table.4 Summary of top three parents and hybrids with respect to their per se performance and combining ability effects for various characters in Indian bean

\begin{tabular}{|c|c|c|c|c|}
\hline \multirow[t]{2}{*}{ Characters } & \multicolumn{2}{|c|}{ Per se performance } & \multicolumn{2}{|c|}{ Combining ability effects } \\
\hline & Parents & $\mathbf{F}_{1} \mathbf{S}$ & GCA & SCA \\
\hline \multirow{3}{*}{$\begin{array}{l}\text { Days to } 50 \% \\
\text { flowering }\end{array}$} & GNIB-21 & GP-kh-120 x Manchhi & GP-167 & GP-kh-120 x Manchhi \\
\hline & GP-kh-120 & GP-167 x GNIB-21 & Manchhi & GP-kh-64 x Kapasi \\
\hline & GP-167 & GP-167 x Kapasi & GNIB-21 & GP-189 x Katargam \\
\hline \multirow{3}{*}{$\begin{array}{l}\text { Days to } \\
\text { maturity }\end{array}$} & GNIB-21 & GP-kh-64 x GNIB-21 & GNIB-21 & GP-1 x Katargam \\
\hline & GP-kh-64 & GP-1 x GNIB-21 & GP-1 & GP-kh-64 x GNIB-21 \\
\hline & GP-167 & GP-167 x GNIB-21 & GP-189 & GP-158 x Manchhi \\
\hline \multirow{3}{*}{$\begin{array}{l}\text { Plant height } \\
\text { (cm) }\end{array}$} & GP-kh-120 & GP-189 x GNIB-21 & GP-189 & GP-189 x GNIB-21 \\
\hline & Katargam & GP-189 X Manchhi & GP-167 & GP-167 x Kapasi \\
\hline & GP-189 & GP-167 x Kapasi & Manchhi & GP-kh-64 x Kapasi \\
\hline \multirow{3}{*}{$\begin{array}{c}\text { Primary } \\
\text { branches per } \\
\text { plant } \\
\end{array}$} & Katargam & GP-kh-64 x Kapasi & GP-kh-120 & GP-kh-64 x Kapasi \\
\hline & Manchhi & GP-kh-120 x Katargam & GP-kh-64 & GP-158 x Manchhi \\
\hline & GP-kh-120 & GP-158 x Manchhi & Manchhi & GP-158 x Katargam \\
\hline \multirow[t]{3}{*}{ Pods per plant } & Katargam & GP-kh-120 x Kapasi & GP-kh-64 & GP-158 x Manchhi \\
\hline & Manchhi & GP-kh-64 x Kapasi & Kapasi & GP-167 x GNIB-21 \\
\hline & GP-kh-120 & GP-kh-64 x Katargam & GP-kh-120 & GP-kh-64 x Katargam \\
\hline \multirow{3}{*}{$\begin{array}{l}\text { Pod length } \\
\text { (mm) }\end{array}$} & GP-189 & GP-189 x Katargam & GP-189 & GP-189 x Katargam \\
\hline & GP-158 & GP-189 x Manchhi & Katargam & GP-kh-64 x Kapasi \\
\hline & Katargam & GP-167 x Katargam & GP-158 & GP-189 x Manchhi \\
\hline \multirow{3}{*}{$\begin{array}{l}\text { Pod width } \\
\text { (mm) }\end{array}$} & GP-158 & GP-167 x Katargam & GP-158 & GP-167 x Katargam \\
\hline & GP-1 & GP-1 x Manchhi & Manchhi & GP-158 x Kapasi \\
\hline & Katargam & GP-189 x Manchh & GP-167 & GP-189 x Katargam \\
\hline \multirow{3}{*}{$\begin{array}{l}\text { Pod weight } \\
\text { (g) }\end{array}$} & GP-158 & GP-158 x Kapasi & GP-158 & GP-158 x Kapasi \\
\hline & Katargam & GP-167 x Katargam & GP-189 & GP-kh-120 x Katargam \\
\hline & GP-189 & GP-167 x Kapasi & Katargam & GP-kh-64 x GNIB-21 \\
\hline \multirow[t]{3}{*}{ Seeds per pod } & GP-1 & GP-158 x GNIB-21 & GP-158 & GP-189 x Kapasi \\
\hline & GP-167 & GP-189 x Kapasi & GNIB-21 & GP-158 x GNIB-21 \\
\hline & Manchhi & GP-1 x GNIB-21 & GP-1 & GP-1 x Katargam \\
\hline \multirow{3}{*}{$\begin{array}{c}\text { Green pod } \\
\text { yield perplant } \\
(\mathrm{g}) \\
\end{array}$} & GP-167 & GP-167 x Katargam & GP-167 & GP-167 x Katargam \\
\hline & GP-189 & GP-167 x GNIB-21 & GP-189 & GP-kh-120 x GNIB-21 \\
\hline & Katargam & GP-189 x GNIB-21 & GNIB-21 & GP-kh-64 x Kapasi \\
\hline \multirow{3}{*}{$\begin{array}{l}\text { Seed yield per } \\
\text { plant } \\
\text { (g) }\end{array}$} & Katargam & GP-kh-64 x Kapasi & Kapasi & GP-158 x Manchhi \\
\hline & GP-189 & GP-kh-120 x Kataragam & Katargam & GP-kh-64 x Kapasi \\
\hline & GP-158 & GP-158 x Manchhi & GP-kh-64 & GP-kh-120 x Katargam \\
\hline \multirow{3}{*}{$\begin{array}{c}\text { Harvest index } \\
(\%)\end{array}$} & GNIB-21 & GP-kh-64 x Katargam & GP-1 & GP-kh-64 x Katargam \\
\hline & Katargam & GP-kh-120 x Kapasi & GP-kh-120 & GP-1 x GNIB-21 \\
\hline & GP-1 & GP-kh-120 x Katargam & Kapasi & GP-167 x Manchhi \\
\hline \multirow{3}{*}{$\begin{array}{l}\text { Straw yield } \\
\text { (g) }\end{array}$} & GP-158 & GP-158 x Katargam & GP-189 & GP-kh-64 x Kapasi \\
\hline & GP-189 & GP-189 x Katargam & GP-167 & GP-167 x GNIB-21 \\
\hline & Kapasi & GP-189 x Manchhi & Katargam & GP-kh-120 x Manchhi \\
\hline \multirow{3}{*}{$\begin{array}{l}100 \text { dry seed } \\
\text { weight }(\mathrm{g})\end{array}$} & GP-158 & GP-167 x Katargam & GP-158 & GP-167 x Katargam \\
\hline & GP-167 & GP-189 x Manchhi & GP-167 & GP-189 x Manchhi \\
\hline & Katargam & GP-158 x Katargam & Katargam & GP-158 x Manchhi \\
\hline \multirow{3}{*}{$\begin{array}{l}\text { Protein content } \\
(\%)\end{array}$} & GNIB-21 & GP-kh-64 x GNIB-21 & GP-kh-64 & GP-kh-120 x Katargam \\
\hline & Kapasi & GP-158 x GNIB-21 & GNIB-21 & GP-1 x Manchhi \\
\hline & GP-1 & GP-189 $\times$ Kapasi & GP-189 & GP-189 x Kapasi \\
\hline
\end{tabular}

A comparative study of the most promising hybrids having high sca effect for green pod yield per plant and for various characters along with gca effects of the parents revealed that the best performing parents may not always be a best general combiners. Significant and high sca effect in desirable direction was observed for days to maturity in the crosses GP-1 x Katargam (-9.875), GNIB21 x GP-kh-64 (-7.292) and GP-158 x Manchhi (-3.819); for plant height in the crosses GNIB-21 x GP-189 (13.432), Kapasi x GP-167 (8.821) and Kapasi x GP-kh-64 (8.704); for primary branches per plant in the 
cross Kapasi x GP-kh-64 (0.542); for pods per plant in the crosses GP-158 $x$ Manchhi (49.011), GNIB-21 GP-167 (44.250) and GPkh-64 x Katargam (32.811); for pod length in the crosses GP-189 x Katargam (9.800), Kapasi x GP-kh-64 (6.978) and GP-189 x Manchhi (6.211); for pod width in the crosses GP-167 x Katargam (2.614), Kapasi x GP158 (2.097) and GP-189 x Katargam (1.864); for pod weight in the crosses Kapasi x GP158 (0.893), GP-kh-120 x Katargam (0.459) and GNIB-21 x GP-kh-64 (0.296); for seeds per pod in the cross Kapasi x GP-189 (0.464).

The cross combination GP-167 x Katargam (10.317) manifested significant sca effect in desirable direction for green pod yield per plant; GP-158 x Manchhi (38.083), Kapasi x GP-kh-64 (24.703) and GP-kh-120 x Katargam (26.008) for seed yield per plant; GP-kh-64 x Katargam (9.139), GNIB-21 x GP-1 (7.274) and GP-167 x Manchhi (6.596) for harvest index; Kapasi $x$ GP-kh-64 (56.732), GNIB-21 x GP-167 (56.295) and GP-kh-120 x Manchhi (55.437) for straw yield; GP-167 x Katargam (7.531), GP-189 x Manchhi (5.781) and GNIB-21 x GP-158 (2.692) for 100 dry seed weight. For majority of the yield attributes the genotypes Kapasi, Katargam, GNIB-21 and GP-167 are involved for producing top ranking hybrids in relation to per se performance and sca effects. The cross combination Kapasi $x$ GP-kh-64 exhibited highest mean for green pod yield per plant followed by GP-kh-120 x Katargam and GP-158 x Manchhi. The cross Kapasi x GP-kh-64 possessed significant sca effects in desirable direction for days to flowering, plant height, primary branches per plant, pod length, green pod yield per plant, seed yield per plant and straw yield.

Whereas, the cross GP-158 x Manchhi had significant sca effects for days to maturity, primary branches per plant, pods per plant and seed yield per plant. General combining ability of these parents were reflected in their hybrids in terms of higher per se performance and sca effects for most of the traits. A perusal of these observations revealed that genotypes GNIB-21, GP-167, GP-kh-64 and GP-kh-120 were early flowering. The worth of these genotypes was reflected in estimates of combining ability. The hybrid GNIB-21 $\mathrm{x}$ GP-167 was best hybrid in relation to per se performance and sca effect considering earliness for flowering and maturity. For days to maturity, top three crosses had either GNIB-21 or GP-167 as one of the parents.

As non-additive gene action was predominant for most of the traits and due to nonfeasibility of hybrid variety in Indian bean, diallel selective mating system may be adopted followed by some sort of biparental mating and recurrent selection for improvement of these traits. Considering genetic improvement of Indian bean, recently, harvest index, photo-insensitivity, pole type plant stature and determinate habit is getting attention in relation to crop geometry. Recombinant Inbred Lines (RILs) may be developed from these hybrids and QTL analysis for pod quality traits, protein content and harvest index may be carried out. Saturated linkage maps may be developed utilizing recent Next Generation Sequencing (NGS) platforms / SNP discovery methods and segregating generations of above hybrids followed by QTL analysis and association mapping.

\section{References}

Aruachalam, V. (1974). Fallacy behind the use of modified line $\mathrm{x}$ tester design. Indian Journal of Genetics and Plant Breeding, 34: 280-287.

Bagade, A. B. (2001). Heterosis and combining ability in Indian bean [Dolichos lablab (L.)]. Unpublished 
thesis, M. Sc. (Agri.), submitted to G.A.U., S. K. Nagar.

Bagade, A. B., Patel, D. U. and Mali, S. C. (2003). Combining ability studies in Indian bean [Dolichos lablab (L.)]. Annals of Plant Physiology, 16 (2): 187190.

Das, I., Seth, T., Durwas, S. V., Dutta, S., Chattopadhyay, A. and Singh B. (2014). Gene action and combining ability for yield and yield component traits in dolichos bean (Dolichos lablab var. typicus). SABRAO Journal of Breeding and Genetics, 46 (2): 293-304.

Jain, H. K. (1975). Development of high yielding varieties of pulses, perspectives, possibilities and experimental approaches. Int. Workshop on grain legumes. ICRISAT, Hyderabad, pp. 177-185.

Kay, D. E. (1973). Food Legumes. Crop and Product Digest No.3. Tropical Product Institute, London. pp. 303.

Kempthorne, O. (1957). An introduction to genetic statistics, John Wiley and sons, New York, pp. 458-471.

Kumar, P. S., Rai, N. H., Singh, D. V. and Rai, K. K. (2015). Genetic analysis for quantitative traits in Indian bean (Lablab purpureus). Indian Journal of Agricultural Sciences, 85(3): 00-00.

Modha, K. G., Kathiria, K. B. and Tukadiya, A. R. (2007). Combining ability analysis for pod yield and its related traits in Indian bean (Lablab purpureus var. lignosus). Veg. Sci., 34(1): 89 -91.

Panse, V. G. and Sukhatme, P. V. (1978). Statistical methods for agricultural workers. I.C.A.R., New Delhi.

Patil, A. B., Desai, D. T., Patil, S. A. and Ghodke, U. R. (2013). Diallel analysis for pod yield and its components traits in vegetable Indian bean (Dolichos lablab L.). African Jounral of Agril. Res., 81(4): 1229-1232.

Rahman, Fatima, K. and Newaz, M. A. (2009). Genetic study and gene action of edible pod yield in $6 \times 6 F_{2}$ diallel population of lablab bean [Lablab purpureus (L.)]. Int. J. Agric. Environ. \& Biotech, 2 (3): 222-226.

Srinivasan, C. and Das, L. D. V. (1996). Combining ability in fodder lablab. Madras Agric. J., 83 (20): 621-622.

Tukadiya, A. R., Kathiria, K. B. and Modha, K. G. (2006). Genetic components analysis for pod yield and its related traits in Indian bean (Lablab purpureus var. typicus). Veg Sci., 33(2): 183-184

Valu, M. G., Pandya, H. M., Dhaduk, H. L. and Vadodaria, M. A. (1999). Combining ability in Indian bean [Lablab purpureus (L.) sweet]. G.A.U. Res. J., 24 (2): 39-42.

Vashi, R. D., Prajapati, R. M. and Vashi, P. S. (2001). Genetic analysis of yield and yield components over environments in Indian bean [Lablab purpureus (L.)]. G.A.U. Res. J., 26 (2): 23-28.

\section{How to cite this article:}

Shreya Sen, R.L. Patel, R.A. Patel, K.G. Modha and Patel, R.K. 2018. Analysis of Combining Ability and Gene Action in Indian Bean [Lablab purpureus (L.) Sweet]. Int.J.Curr.Microbiol.App.Sci. 7(04): 1790-1798. doi: https://doi.org/10.20546/ijcmas.2018.704.203 